\title{
Rate dependence of electrical and mechanical properties of conductive polymer nanocomposites
}

\author{
J.R. Foley ${ }^{1}$, C.L. Stilson ${ }^{1}$, K.K.G. Smith ${ }^{1,2}$, C.M. McKinion ${ }^{1,3}$, C. Chen ${ }^{4}$, S. Ganguli ${ }^{4}$, and A.K. Roy ${ }^{4}$ \\ ${ }^{1}$ Air Force Research Laboratory, Eglin AFB, FL, USA \\ ${ }^{2}$ National Research Council Postdoctoral Research Associate, Eglin AFB, FL, USA \\ ${ }^{3}$ Doolittle Institute, Fort Walton Beach, FL, USA \\ ${ }^{4}$ Air Force Research Laboratory, Wright-Patterson AFB, OH, USA
}

\begin{abstract}
Conductive polymer nanocomposites with enhanced electrical and thermal properties show promise as an alternative solution for electronic materials. For example, electronic interconnect materials will have comparable electrical and thermal conductivity to solder with an increased operating range of strain and temperature. This paper documents the fabrication and experimental evaluation of a prototype conductive polymer nanocomposite. Material selection, fabrication processes, and initial characterization of a low Tg polymer with a high fill ratio of carbon nanotubes is presented. The electrical and thermal properties of the composite are measured and compared with predictions. The mechanical properties are measured using dynamic mechanical analysis (DMA) over a wide temperature range. The mechanical and electrical responses of the conductive polymer composite are simultaneously measured at higher strain rates using a modified split Hopkinson pressure bar (SHPB) apparatus. The dynamic stress-strain response is obtained using traditional analytic methods (e.g., two- and three-wave analysis). The electrical response is observed using constant current excitation with high bandwidth $(>500 \mathrm{kHz})$ instrumentation. The dynamic compression data implies the change in electrical resistance is solely a function of the material deformation, i.e., the material exhibits constant electrical conductivity and is insensitive to the applied loads. DMA and SHPB dynamic data are used to estimate the parameters in a Mulliken-Boyce constitutive model, and the resulting behavior is critically evaluated. Finally, progress towards improving the polymer composite's mechanical, electrical, and thermal properties are discussed.
\end{abstract}

\section{Introduction}

The global electronics industry is dominated by components designed for commercial electronics [1]. Accordingly, the design architecture, fabrication process, materials, component design, and testing [2] all reflect the mass-production, commercial-off-the-shelf (COTS) nature of consumer electronics. However, these same processes, materials, etc., are often used in electronics subjected to much harsher operating environments. The corresponding failure modes are diverse and depend on the geometry, materials, and physics of operation of the devices [3]. For example, Fig. 1 shows an SEM image of the cross-section of a surface mount resistor subjected to severe mechanical shock environments [4,5]. The mechanical loading initiated a crack that has propagated along the interface between the fiber-reinforced polymer printed circuit board and the copper trace material. The particular damage mechanism, known as pad lift-off [6] or pad cratering, occurs at the interconnect between the assembly and the component. Other electronics failure modes at device interconnects include solder joint fracture, component termination failures, or component stress cracking.

Developing alternatives for the packaging and assembly of traditional electronics requires understanding of the multifunctional response of candidate materials. This paper documents the fabrication and initial characterization of the combined thermal, electrical, and

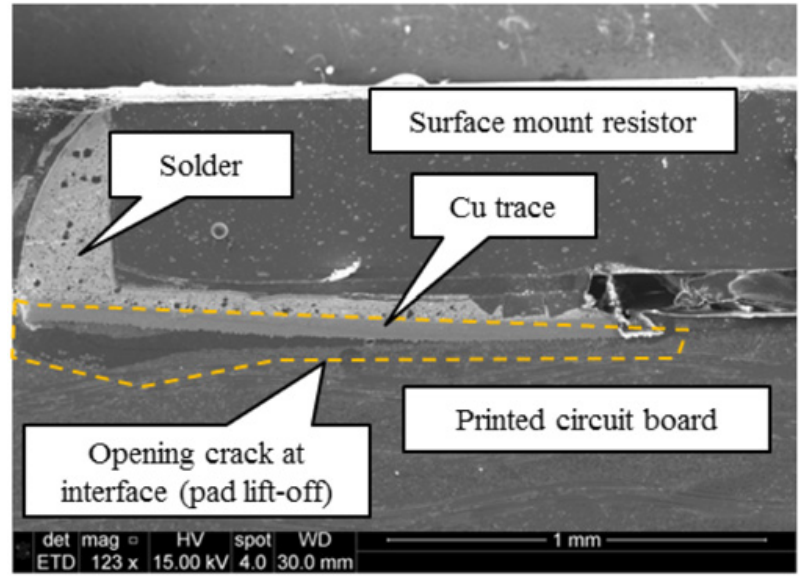

Figure 1. SEM image of a surface-mount electronic component cross-section after high shock loading. The dashed line indicates the onset of pad lift-off at the printed circuit board-copper trace interface.

rate-dependent mechanical response of a new electricallyconductive polymer composite.

\section{Material development}

A conductive polymer nanocomposite $(\mathrm{CPnC})$ was proposed as an alternative material for electronic 
Table 1. Design objectives for the conductive polymer nanocomposite in comparison to $\mathrm{Sn}-37 \mathrm{~Pb}$ and $\mathrm{SAC} 305$ solders.

\begin{tabular}{|l|l|l|l|l|l|}
\hline $\begin{array}{l}\text { Design } \\
\text { Metric }\end{array}$ & Sym. & $\begin{array}{l}\text { Sn- } \\
\mathbf{3 7 P b}\end{array}$ & $\begin{array}{l}\text { SAC- } \\
\mathbf{3 0 5}\end{array}$ & $\begin{array}{l}\text { CPnC } \\
\text { Goal }\end{array}$ & Units \\
\hline $\begin{array}{l}\text { Electrical } \\
\text { resistivity }\end{array}$ & $\rho$ & 0.146 & 0.132 & $\leq 0.5$ & $\mu \Omega \cdot \mathrm{m}$ \\
\hline $\begin{array}{l}\text { Thermal } \\
\text { conductivity }\end{array}$ & $\kappa$ & 50.9 & 58 & $\geq 58$ & $\mathrm{~W} / \mathrm{m} \cdot \mathrm{K}$ \\
\hline $\begin{array}{l}\text { Tensile } \\
\text { Elongation }\end{array}$ & $\Delta L / L_{0}$ & $\sim 50$ & 35 & $>100$ & $\%$ \\
\hline $\begin{array}{l}\text { Glass Trans. } \\
\text { Temp. }\end{array}$ & $T_{g}$ & N/A* & N/A & $<273$ & $\mathrm{~K}$ \\
\hline Viscosity & $\eta$ & N/A & N/A & $<1 \mathrm{e} 6$ & poise \\
\hline
\end{tabular}

* Note: glass transition temperature and viscosity are not meaningful for solder alloys except during the reflow process and are therefore not applicable.

interconnects. This section reviews the design objectives, composition and fabrication process of the initial composite, and characterization of the material.

\subsection{Material performance objectives}

The primary performance objective for the $\mathrm{CPnC}$ is to reproduce the thermal and electrical properties of solder while exhibiting strain-tolerance over a wide range of operating conditions. A eutectic lead-based solder alloy, $\mathrm{Sn}-37 \mathrm{~Pb}(63 \% \mathrm{Sn}, 37 \% \mathrm{~Pb})$, and a lead-free solder alloy, SAC305 (96.5\% Sn, 3\% Ag, 0.5\% Cu), were chosen as references [7]. The strain tolerance was given indicated by the strain to failure (i.e., ultimate strain). Viscosity, particularly shear-thinning behavior, was sought to aid in mixing, processing, and ultimately enable manufacturing concepts. Finally, the electrical properties should be insensitive to strain (i.e., not change resistivity as a function of deformation). Table 1 below lists the design objectives for the study.

\subsection{Fabrication process}

A low $T_{g}$ polymer matrix was chosen instead such that the material would remain compliant during dynamic events and extend the ultimate strain. The polymer, Epon 828 with Jeffamine D2000 hardener, has relatively poor electrical and thermal performance (i.e., conductivity) in the cured state. In order to improve the electrical and thermal conductivity in a manner similar to commercially available conductive epoxies (e.g., the Ag-epoxy examined in [8]), conductive nanoparticles were considered as the filler. Candidate fillers included Ag nanoparticles and multiwalled carbon nanotubes (MWCNT).

Initial compositions investigated included two heavily filled CPnC's. The first consisted of $20 \%$ (by weight) $\mathrm{Ag}$ nanoparticles, $10 \%$ MWCNT and the Epon 828/D2000 matrix. The second was $20 \%$ MWCNT with the Epon $828 / \mathrm{D} 2000$ matrix. The processing procedure involves three mixing cycles of $2000 \mathrm{rpm}$ for 3 minutes each in a Thinky planetary centrifugal mixer. After each mix cycle, the composite material is degassed under vacuum (20 kPa) while continuing mixing for 2 minutes. After three mixing cycles, the mixture is cast in either silicone or Teflon

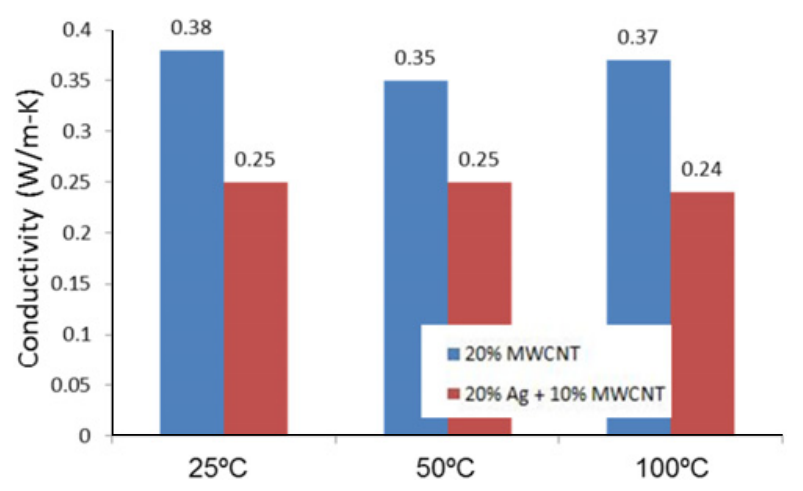

Figure 2. Thermal conductivity at three different temperatures.

molds, placed in vacuum bags, and subsequently cured in an autoclave. The curing temperature profile involves (1) a ramp from room temperature to $120^{\circ} \mathrm{C}$ at a rate of $1{ }^{\circ} \mathrm{C} / \mathrm{min}$., (2) hold at $120^{\circ} \mathrm{C}$ for 3 hours, then (3) a cool down to room temperature; pressure is maintained at $690 \mathrm{kPa}$ and a vacuum of $-100 \mathrm{kPa}$ (gauge pressure) is applied to the parts during curing. The vacuum and pressure are released after the parts cooled and the samples then separated from the molds.

\subsection{Characterization}

After fabrication of the samples, basic thermal, electrical, and mechanical charaterization of the candidate CPnC's was performed. Electrical resistivity was estimated using the van der Pauw technique. The 20\% MWCNT/Epon $828 / \mathrm{D}-2000$ prototype material was measured to have an electrical resistivity of $0.5 \mu \Omega \cdot \mathrm{m}$, reaching the design goal. The $20 \%$ Ag/10\% MWCNT/Epon 828/D2000 was observed to have a much higher resistivity,

The thermal conductivity was measured using laser flash analysis. The 20\% MWCNT/Epon 828/D2000 and the 20\% Ag/10\% MWCNT/Epon 828/D2000 were measured at $25^{\circ} \mathrm{C}, 50{ }^{\circ} \mathrm{C}$, and $100^{\circ} \mathrm{C}$. The results are shown in Fig. 2 below. While both conductivities were significantly below the design goal, the $20 \%$ MWCNT material showed higher average thermal conductivity $(0.37 \mathrm{~W} / \mathrm{m} \cdot \mathrm{K})$ than the $20 \% \mathrm{Ag} / 10 \%$ MWCNT composite $(0.25 \mathrm{~W} / \mathrm{m} \cdot \mathrm{K})$.

One of the key unanswered questions revolves around the microstructure, specifically whether the nanoparticles were uniformly distributed throughout the matrix and the nature the interfaces between nanoparticles. SEM and TEM analyses were performed; images from the SEM analysis on the 20\% Ag/10\% MWCNT/Epon 828/D2000 and the 20\% MWCNT/Epon 828/D2000 are shown in Fig. 3 and Fig. 4, respectively. The $20 \%$ mass loading of the silver nanoparticle flakes was initially expected to contribute strongly to the enhanced transport properties within the material. However, Fig. 3 illustrates that the very dense $\mathrm{Ag}$ is sparsely distributed and does not enhance the overall network due to the isolated location of each particle, explaining the lower thermal conductivity and higher electrical resistivity. Commercial conductive epoxies achieve enhanced transport through much higher mass loadings, typically $65 \%$ or higher [9]. Additionally, there were few observable interfaces between the two types 


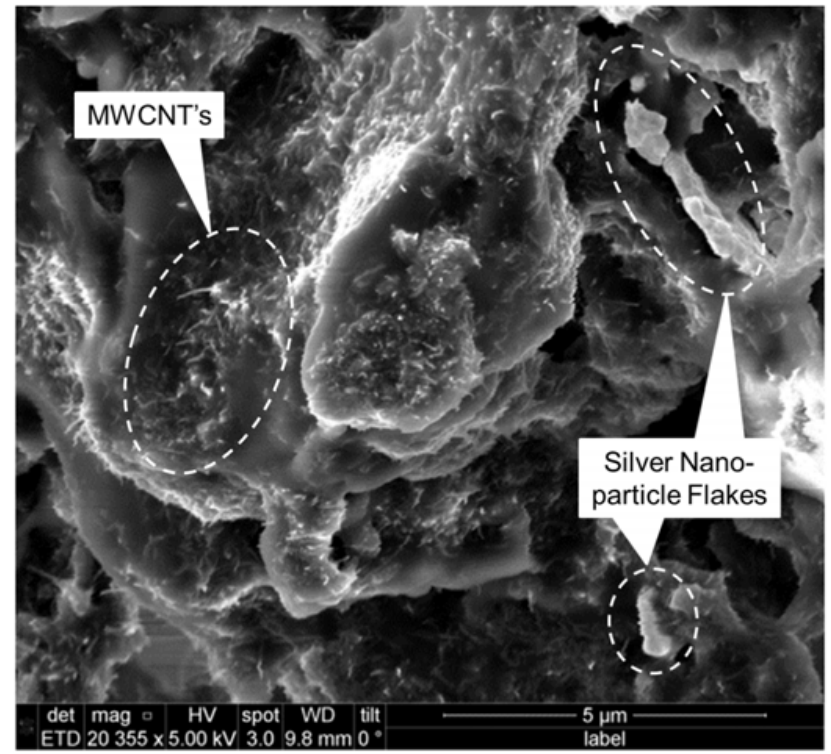

Figure 3. SEM image of the $20 \% \mathrm{Ag} / 10 \%$ MWCNT CPnC showing carbon nanotubes and the flakes of silver nanoparticles (matrix has been removed).

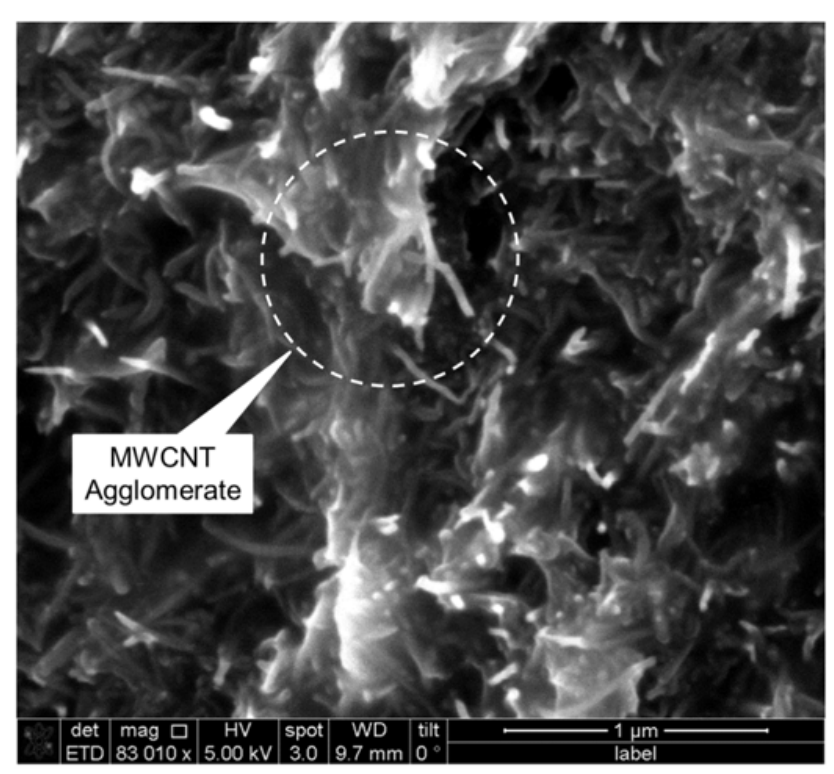

Figure 4. SEM image of the $20 \%$ MWCNT CPnC showing multiwalled carbon nanotubes with significant agglomeration (matrix material has been removed).

of nanoparticle. The MWCNT-only CPnC, shown in Fig. 4, exhibits slightly enhanced transport relative to the silverloaded CPnC, presumably through conduction within the agglomerated nanotubes.

The overall better performance of the $20 \%$ MWCNT composite-with a lower electrical resistivity and higher thermal conductivity-led to its selection as the best candidate for more detailed analysis. Due to the importance of processability for electronics materials, the viscosity of the $\mathrm{CPnC}$ was measured as a function of curing temperature profile, shown in Fig. 5. The melt-thinning

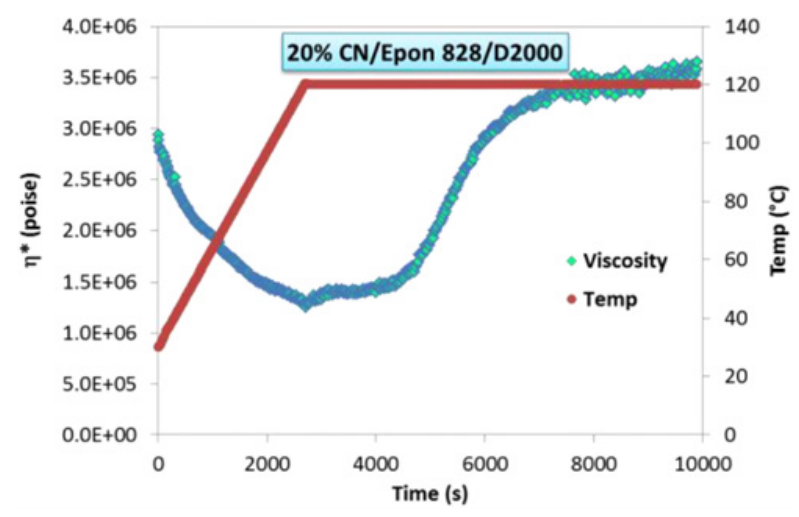

Figure 5. Viscosity and temperature vs. time for the $20 \%$ MWCNT CPnC.

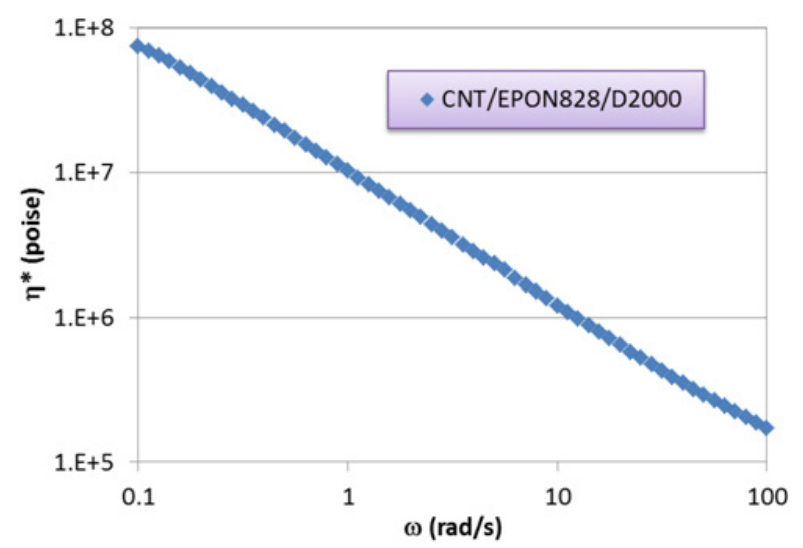

Figure 6. Shear-thinning behavior of the $20 \%$ MWCNT CPnC.

of the matrix contributes to the reduced viscosity through the temperature, reaching a minimum of approximately $1.3 \mathrm{e} 6$ poise at the cure temperature. The shear-thinning behavior of the composite is shown in Fig. 6; the 20\% MWCNT material exhibits significant shear-thinning, which is desirable for many processing techniques.

\subsection{Dynamic mechanical analysis}

Dynamic mechanical analysis (DMA) [10] was then performed on both candidate CPnC's. DMA samples of $17.9 \mathrm{~mm}$ long, $11 \mathrm{~mm}$ wide, and $3.1 \mathrm{~mm}$ thick were cast for each of the candidate materials and the matrix material. The samples were tested in a single cantilever configuration in a TA Instruments Q800 [11,12]. The excitation frequency was set at $1 \mathrm{~Hz}$ and the samples were ramped through the temperature range of $-120^{\circ} \mathrm{C}$ to $+120^{\circ} \mathrm{C}$ at a rate of $3{ }^{\circ} \mathrm{C} /$ minute. The displacement was held constant at $20 \mu \mathrm{m}$ for this analysis.

Typical DMA data for the CPnC's is shown in Fig. 7 at the measured frequency of $1 \mathrm{~Hz}$. The low $T_{g}$ is readily apparent, and the room temperature $(298 \mathrm{~K})$ modulus of both candidates are approximately $70 \mathrm{MPa}$. The frequency can be converted to an equivalent strain rate $(\dot{\varepsilon})$ using the equivalence relationship [13],

$$
\dot{\varepsilon}=4 \omega d_{0} / l_{g},
$$

where $\omega$ is the angular frequency (in $\mathrm{rad} / \mathrm{s}$ ), $d_{0}$ is the amplitude of the displacement, and $l_{g}$ is the specimen gage 


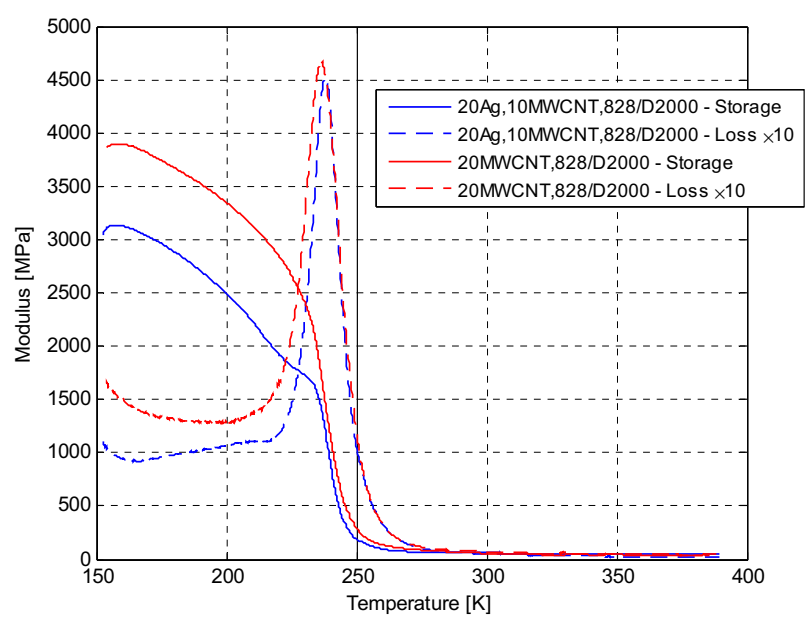

Figure 7. DMA storage and loss modulus data for two candidate CPnC's.

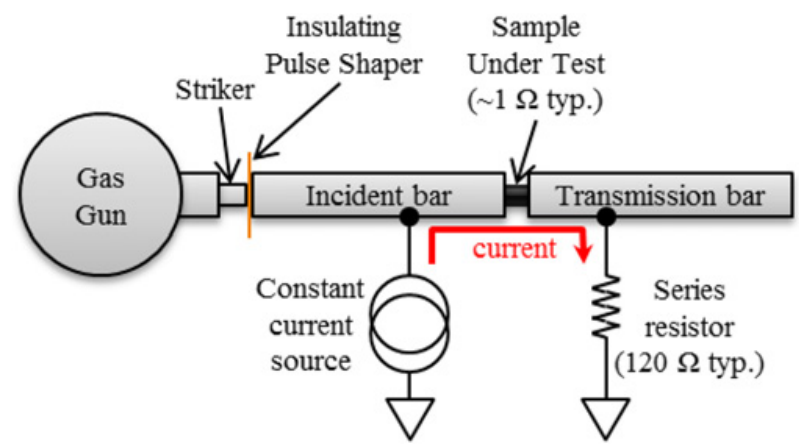

Figure 8. Schematic diagram of modified SHPB apparatus used to simultaneously monitor mechanical and electrical response of the sample under test.

length. For this sample geometry, the equivalent strain rate is $\dot{\varepsilon} \approx 0.03 \mathrm{~s}^{-1}$.

\section{Dynamic compression experiments}

\subsection{Apparatus}

A split Hopkinson pressure bar (SHPB) apparatus [14] was modified to allow simultaneous measurement of the electrical resistance of the sample by using the incident and transmission bars as electrodes. A schematic is shown in Fig. 8. 6 foot $(1.81 \mathrm{~m})$ long aluminum alloy (6061) bars with 1 in. $(25.4 \mathrm{~mm})$ diameter were used for both the incident and transmission bar.

The technique to simultaneously measure the electrical and mechanical response of the material is most similar to previous work by Heeder et al. [15]. The primary distinction is the much higher mass loading of the $20 \%$ MWCNT CPnC investigated in this work. Other recent studies have attempted similar measurements but on much higher equivalent resistance composites $[16,17]$.

A Precision Filters 28144 constant current signal conditioner [18] is used to provide the excitation current. Electrical connection to the incident and transmission bars is made using hose clamps compressing a copper mesh (to which the wires are soldered) as shown in Figure. Due to the low resistivity of the nanocomposite (the sample

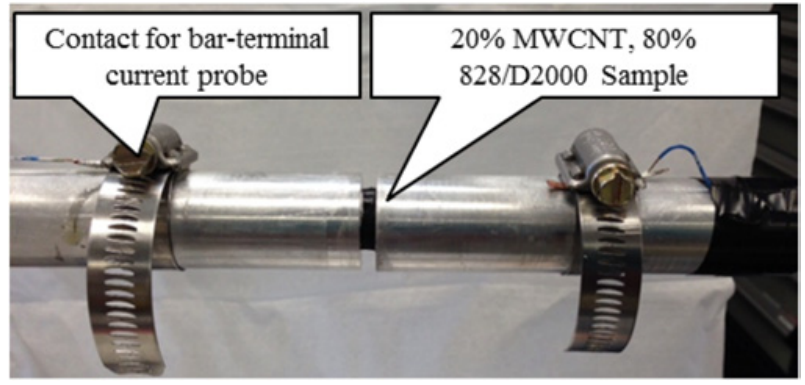

Figure 9. Photographic detail of SHPB experiment showing sample and contact configuration.

resistance is typically less than $1 \Omega$ ), a series resistor is necessary to raise the total resistance to the expected range of a strain gage (in this case, approximately $120 \Omega$ ). Uncured conductive epoxy is used in lieu of conductive grease as a multifunctional surface finish: it reduces friction at the sample-bar interfaces and also reduces the contact resistance.

Dynamic strain is measured on the incident and transmission bars using Kyowa semiconductor strain gages [19]; the same Precision Filters system used to measure the sample resistance provides the strain gage conditioning. Both sensor and sample resistance signals are digitized by a National Instruments data acquisition system with PXI-6133 cards [20].

\subsection{Sample material, preparation, and geometry}

The 20\% MWCNT nanocomposite samples were prepared as noted in Sect. 2.2 and cast into $0.625 \mathrm{in}$. (16 mm) diameter rods. SHPB sample disks with variable lengths between 0.125 in. $(3 \mathrm{~mm})$ and $0.188 \mathrm{in} .(4.8 \mathrm{~mm})$ were cut from these rod.

\subsection{Results and discussion}

As expected with a low $T_{g}$ matrix, the $20 \%$ MWCNT $\mathrm{CPnC}$ exhibited a compliant, rubbery response as shown in the stress-strain curve shown in Fig. 10 with a measured strain rate of $522 \mathrm{~s}^{-1}$. The material exhibited excellent recovery, exhibiting no measurable change in thickness after dynamic compression and no discernible damage.

The electrical response of the material in various tests is shown as a function of time is shown in Fig. 11 and Fig. 12. The bulk resistivity of the sample was initially hypothesized to follow the compressive stress (or pressure) due to the MWCNT networks acquiring more contact points, decreasing the overall composite material's resistance. However, as Fig. 11 shows, there was no direct correlation to support this hypothesis. The opposite scenario, where the bulk resistivity $\left(\rho_{0}\right)$ is constant, would yield a scenario where the change in resistance $(\Delta R)$ follows from the geometric distortion of the sample,

$$
\Delta R=\rho_{0} \Delta(L / A),
$$

where $L$ and $A$ are the instantaneous length and area of the conductive path in the sample. Assuming full participation and one-dimensional compression, this result would be proportional to the net compression of the sample. This 


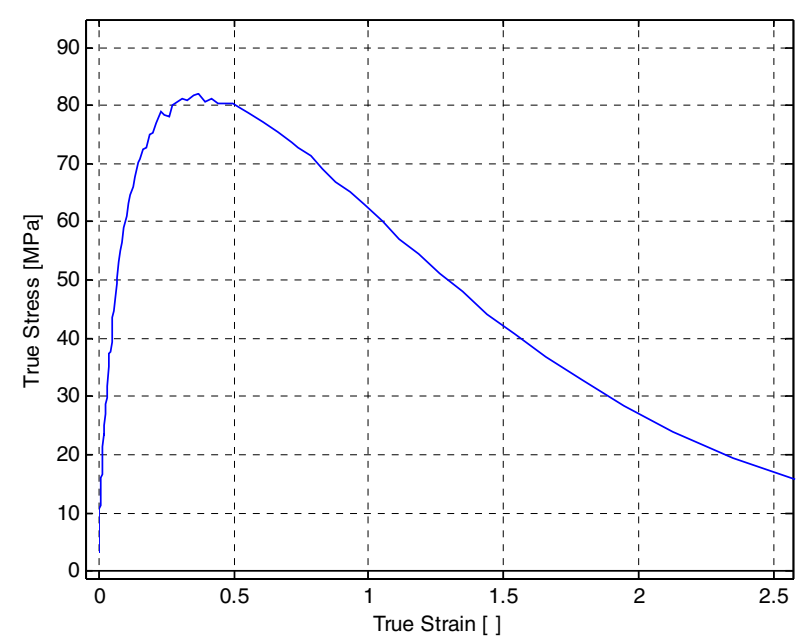

Figure 10. Typical stress-strain diagram for the 20\% MWCNT CPnC.

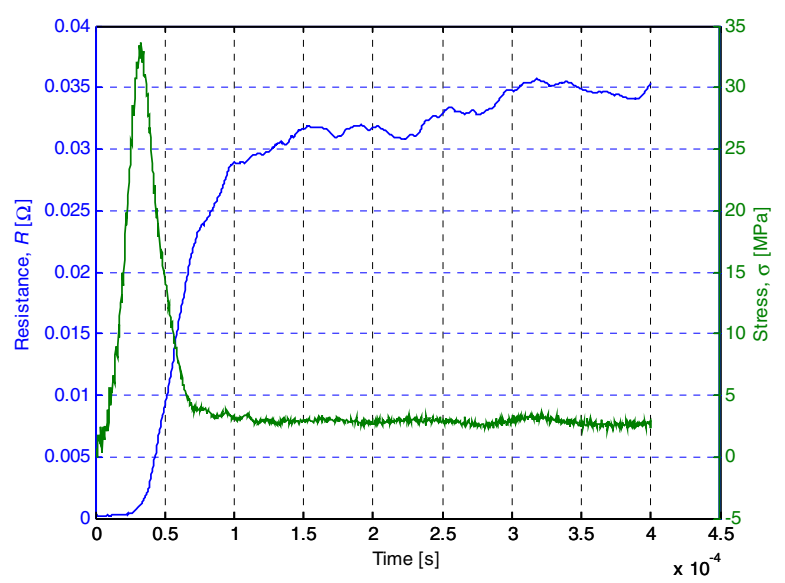

Figure 11. Change in resistance and stress vs. time for the nanocomposite.

proportionality is evident in Fig. 12, supporting this conclusion.

\subsection{Constitutive analysis}

The Ogden model [21] was initially considered due to the rubbery nature of the nanocomposite, however the calcualted compression of the $\mathrm{CPnC}$ with minimal radial distortion suggested this was inappropriate.

The Mulliken-Boyce (M-B) constitutive model was then chosen for analyzing the data based on previous work [22,23]. The M-B model [24,25] is a two phase ( $\alpha$ and $\beta$ ) viscoelastic-viscoplastic model for glassy polymers that incorporates both polymer network stress and the chain stresses. The model has been shown to accurately reproduce rate-dependent behavior of polymers. DMA data [26] is used to estimate the activation energies, mean temperatures, and shift factors associated with different phases of a polymeric material associated in the M-B model. However, the CPnC's considered are well above the glass transition temperature. Nonetheless, the model was implemented with the assumption that the nanotube networks can be modeled by the chain stresses

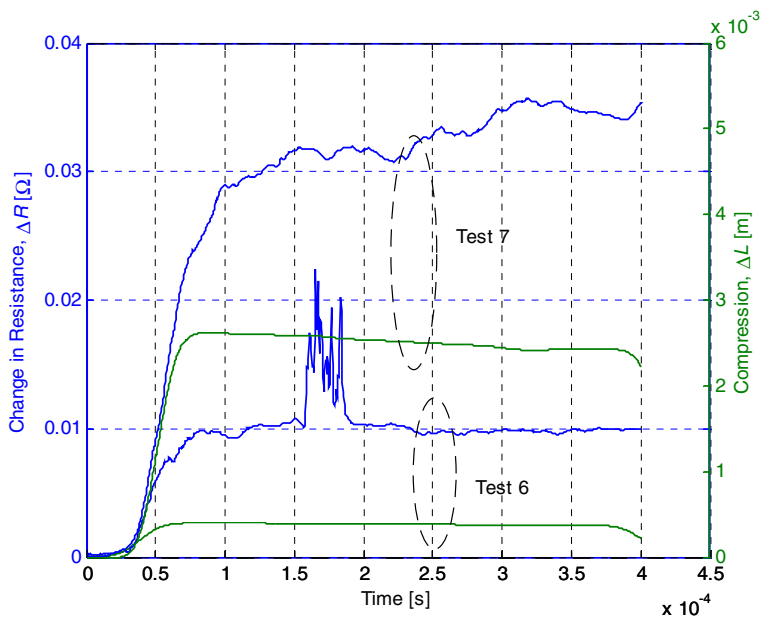

Figure 12. Resistance change (left axis) and total deformation (right axis) for two tests. The roughly proportional response implies little change in the bulk resistivity as a function of pressure. (An intermittent electrical contact is evident in the resistance during test 6. .)

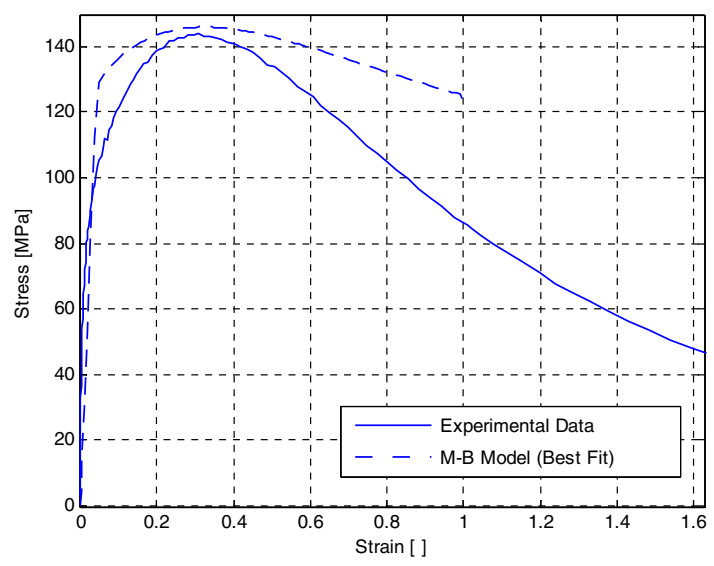

Figure 13. Overlay of expermental and Mulliken-Boyce model stress-strain diagrams for a $20 \%$ MWCNT CPnC sample.

analagous to the polymer (quantified as a Langevin spring element in the viscoelastic model).

A genetic algorithm was implemented in Matlab [27] to estimate the M-B model parameters. The DMA data was used for the modulus as a function of temperature with the $\beta$ phase assumed to be negligible. A resulting fit is shown in Fig. 13. The rubbery modulus was estimated to be $20 \mathrm{MPa}$ with a stretch factor $(N)$ of 2.9 , indicating significant participation in the network stress term. The estimates for the phase activation energies $\left(\sim 10^{-19} \mathrm{~J}\right)$ nor attempt frequencies $\left(\sim 10^{19} \mathrm{~s}^{-1}\right)$ are not expected to be accurate due to the system being above the glass transition temperature. Overall, the parameter estimates were limited by the lack of shift factors associated with the DMA (i.e., no frequency-dependent information), variability between dynamic stress-strain data sets, and lack of dynamic data at multiple strain rates. A more appropriate constitutive model should also be sought, given the inappropriate use of the M-B model in a solely rubbery phase. 


\section{Future work}

The development of the $\mathrm{CPnC}$ is at a very immature stage. While the initial results are promising, the thermal conductivity and electrical resistivity need to be significantly improved. CNT interface functionalization (metallic approach) is being pursued as a possible means to further improve the properties of the next iteration of the nanocomposite. More thorough examination of the ratedependent effects, including a wider range of frequencies of DMA data and adding both quasi-static and dynamic tensile and compressive testing at different strain rates, are also underway. Improvements to the modified SHPB are also underway, such as using bars with lower impedance (e.g., hollow Al bars) and utilizing longer duration loading pulses. Detailed examination of the coupling between the mechanical and electrical response of the material is also in progress. Lastly, analysis of the stress-strain data using different constitutive models is planned.

\section{Conclusion}

The development of a conductive polymer nanocomposites with enhanced electrical and thermal properties was pursued as an alternative material for electronic interconnects. The material synthesis method and electrical/thermal characterization of two prototype CPnC's compositions$20 \% \mathrm{Ag} / 10 \%$ MWCNT and 20\% MWCNT in Epon 828/D2000 matrix-was presented. Mechanical properties of the resulting rubbery material was measured over a wide temperature range using dynamic mechanical analysis (DMA). The mechanical and electrical responses of the conductive polymer composite were simultaneously measured at higher strain rates using a modified split Hopkinson pressure bar (SHPB) apparatus. The change in electrical resistance of the sample during dynamic deformation is shown to be largely a function of the material deformation. If the bulk resistivity is assumed to be constant, the resulting change in resistance implies the material is largely insensitive to the applied loads. The stress-strain curves were estiamted using a Mulliken-Boyce model with marginal results. Finally, ongoing research towards improving the polymer composite's mechanical, electrical, and thermal properties was discussed.

The authors wish to thank the U.S. Air Force Office of Scientific Research (Project Officer: Dr. Byung-Lip "Les" Lee) for their support of this project. Opinions, interpretations, conclusions, and recommendations are those of the authors and are not necessarily endorsed by the United States Air Force.

\section{References}

[1] W. Custer, IPC Outlook (downloaded from www.ipc.org) (2011)

[2] JEDEC, Board level drop test method of components for handheld electronic products (2003)
[3] R.A. Amy, G.S. Aglietti, and G. Richardson, Shock \& Vibration. 16, 45 (2009)

[4] R.D. Lowe, et al., Proceedings of the 85th Shock and Vibration Symposium (2014)

[5] R.D. Lowe, et al., Proceedings of the 85th Shock and Vibration Symposium (2014)

[6] J.W. Dally, P. Lall, and J.C. Suhling, Mechanical Design of Electronic Systems (College House Enterprises Knoxville, TN, 2008)

[7] T. Siewert, et al., Database for Solder Properties with Emphasis on New Lead-free Solders (National Institute of Standards and Technology Colorado, 2002)

[8] X. Luo and D.D.L. Chung, Journal of Materials Science. 34, 273 (1999)

[9] Tra-Con, Tra-Duct 2902 Room Temperature Conductive Silver Epoxy Adhesive (Technical Product Data and MSDS) (2002)

[10] Introduction to Dynamic Mechanical Analysis (DMA): A Beginner's Guide (PerkinElmer, Inc. Waltham, MA, 2008)

[11] DMA Q800 Specifications (TA Instruments, 2010)

[12] TA Instruments Dynamic Mechanical Analyzer (TA284) (TA Instruments, 2011)

[13] A.D. Mulliken and M.C. Boyce, International Journal of Solids and Structures. 43, 1331 (2006)

[14] G.T. Gray III, in ASM Handbook. Vol 8: Mechanical Testing and Evaluation, edited by H. Kuhn and D. Medlin (ASM International, Materials Park, 2002), pp. 462

[15] N. Heeder, et al., Journal of Materials Science. 47, 3808 (2012)

[16] A.S. Lim, et al., Composites Science and Technology. 71, $616(2011)$

[17] L. Jin-tao, C. Jian-kang, and Z. Ming-hua, Polymers \& Polymer Composites. 20, 45 (2012)

[18] Precision 28144 Quad-Channel Wideband Transducer Conditioner with Voltage and Current Excitation (Datasheet) (Precision Filters, Inc. Ithaca, 2009)

[19] Kyowa KSP-2-120-E4 Strain Gage Datasheet (Kyowa Electronic Instruments Co., 2014)

[20] NI PXI-6133 Specifications. (Available from ni.com) edn. (National Instruments Austin, 2003)

[21] O.A. Shergold, N.A. Fleck, and D. Radford, Int. J. Impact Engng. 32, 1384 (2006)

[22] J. Jordan, J. Foley, and C. Siviour, in Mechanics of Time Dependent Materials (Monterey, CA, 2008)

[23] J. Jordan, J. Foley, and C. Siviour, Mechanics of Time-Dependent Materials. 12, 249 (2008)

[24] A.D. Mulliken. Massachusetts Institute of Technology, 2004

[25] A.D. Mulliken. Massachusetts Institute of Technology, 2006

[26] J.R. Foley, J.L. Jordan, and C.R. Siviour, Proceedings of DYMAT 2012 (2012)

[27] Matlab User's Manual (version 2011b) (Mathworks, 2011) 